Fang Liu, Guo-Kai Jia and Guang-Chuan Ou*

\title{
Crystal structure of (4-(4-chlorophenyl)-5-ethyl-1,3-dioxane-5- carboxylato- $\left.\mathrm{K}^{2} \mathrm{O}, \mathrm{O}^{\prime}\right)-(5,5,7,12,12,14$-hexamethyl-1,4,8,11- tetraazacyclotetradecane- $\left.\mathrm{K}^{4} N, N^{\prime}, N^{\prime \prime}, N^{\prime \prime \prime}\right)$ nickel(II) perchlorate monohydrate, $\mathrm{C}_{29} \mathrm{H}_{52} \mathrm{Cl}_{2} \mathrm{~N}_{4} \mathrm{NiO}_{9}$
}

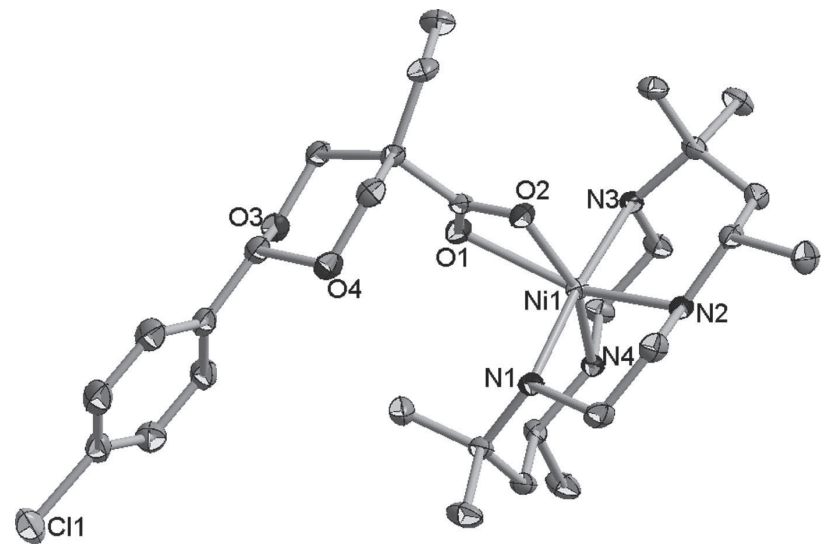

https://doi.org/10.1515/ncrs-2018-0095

Received May 22, 2018; accepted July 26, 2018; available online August 8, 2018

\section{Abstract}

$\mathrm{C}_{29} \mathrm{H}_{52} \mathrm{Cl}_{2} \mathrm{~N}_{4} \mathrm{NiO}_{9}$, monoclinic, $P 2_{1} / n$ (no. 14), $a=$ 10.8347(19) $\AA, b=14.502(3) \AA, c=21.731(4) \AA, \beta=93.344(2)^{\circ}$, $V=3408.6(10) \AA^{3}, Z=4, R_{\mathrm{gt}}(F)=0.0360, w R_{\text {ref }}\left(F^{2}\right)=0.1009$, $T=296(2) \mathrm{K}$.

\section{CCDC no.: 1569959}

The crystal structure is shown in the figure. Hydrogen atoms are omitted for clarity. Tables 1 and 2 contain details on crystal structure and measurement conditions and a list of the atoms including atomic coordinates and displacement parameters.

\section{Source of material}

An acetonitrile solution $(20 \mathrm{~mL})$ of $[\mathrm{NiL}]\left(\mathrm{ClO}_{4}\right)_{2}(0.270 \mathrm{~g}$, $0.5 \mathrm{mmol}) \quad(\mathrm{L}=5,5,7,12,12,14$-hexamethyl-1,4,8,11-tetraazacyclotetradecane) was added to a solution of 4-(4chlorophenyl)-5-ethyl-1,3-dioxane-5-carboxylic acid (0.270 g, $1.0 \mathrm{mmol})$ and $\mathrm{NaOH}(0.04 \mathrm{~g}, 1.0 \mathrm{mmol})$ in the minimum

*Corresponding author: Guang-Chuan Ou, Department of Biology and Chemistry, Hunan University of Science and Engineering, Yongzhou Hunan 425199, P.R. China, e-mail: ogcouguangchuan@163.com

Fang Liu and Guo-Kai Jia: Department of Biology and Chemistry, Hunan University of Science and Engineering, Yongzhou Hunan 425199, P.R. China
Table 1: Data collection and handling.

\begin{tabular}{ll}
\hline Crystal: & Prism, blue \\
Size: & $0.45 \times 0.42 \times 0.30 \mathrm{~mm}$ \\
Wavelength: & Mo $K \alpha$ radiation $(0.71073 \AA)$ \\
$\mu:$ & $0.78 \mathrm{~mm}^{-1}$ \\
Diffractometer, scan mode: & Bruker SMART, $\varphi$ and $\omega$-scans \\
$\theta_{\max }$, completeness: & $27.5^{\circ},>97 \%\left(\right.$ up to $\left.25.2^{\circ},>99 \%\right)$ \\
$N(h k l)_{\text {measured }}, N(h k l)_{\text {unique }}, R_{\text {int }}:$ & $37399,7623,0.019$ \\
Criterion for $I_{\text {obs }}, N\left(h k l_{\text {gt }}:\right.$ & $I_{\text {obs }}>2 \sigma\left(l_{\text {obs }}\right), 6625$ \\
$N(\text { param })_{\text {refined }}:$ & 419 \\
Programs: & Bruker programs [1], SHELX [2, 3] \\
\hline
\end{tabular}

Table 2: Fractional atomic coordinates and isotropic or equivalent isotropic displacement parameters $\left(\AA^{2}\right)$.

\begin{tabular}{lrrrr}
\hline Atom & $\boldsymbol{x}$ & $\boldsymbol{y}$ & $\boldsymbol{z}$ & $\boldsymbol{U}_{\text {iso }} \boldsymbol{U}_{\text {eq }}$ \\
\hline Ni1 & $0.26359(2)$ & $0.52040(2)$ & $0.16501(2)$ & $0.02486(8)$ \\
Cl1 & $0.82771(6)$ & $0.82560(4)$ & $0.19051(3)$ & $0.05458(16)$ \\
Cl2 & $-0.34104(7)$ & $0.02911(5)$ & $0.00498(4)$ & $0.0705(2)$ \\
N1 & $0.09651(14)$ & $0.51940(11)$ & $0.10828(7)$ & $0.0305(3)$ \\
H1C & 0.1159 & 0.4846 & 0.0714 & $0.037^{*}$ \\
N2 & $0.27869(14)$ & $0.65691(10)$ & $0.13539(7)$ & $0.0295(3)$ \\
H2C & 0.2389 & 0.6962 & 0.1651 & $0.035^{*}$ \\
N3 & $0.42254(14)$ & $0.53193(11)$ & $0.22709(7)$ & $0.0294(3)$ \\
H3A & 0.4565 & 0.4695 & 0.2313 & $0.035^{*}$ \\
N4 & $0.16406(13)$ & $0.53492(10)$ & $0.24460(7)$ & $0.0265(3)$ \\
H4D & 0.1503 & 0.6012 & 0.2499 & $0.032^{*}$ \\
O1 & $0.29848(12)$ & $0.36685(9)$ & $0.16072(6)$ & $0.0344(3)$ \\
O1W & $0.34802(17)$ & $0.24713(12)$ & $0.25914(8)$ & $0.0539(4)$ \\
H1WB & $0.322(2)$ & $0.2800(17)$ & $0.2283(9)$ & $0.065^{*}$ \\
H1WA & $0.4210(13)$ & $0.2635(19)$ & $0.2701(11)$ & $0.065^{*}$ \\
O2 & $0.34195(13)$ & $0.46143(9)$ & $0.08699(6)$ & $0.0351(3)$ \\
O3 & $0.21761(13)$ & $0.18276(10)$ & $0.08573(6)$ & $0.0397(3)$ \\
O4 & $0.17587(14)$ & $0.28523(10)$ & $0.00549(6)$ & $0.0389(3)$ \\
O5 & $0.7527(2)$ & $0.8441(2)$ & $0.23994(11)$ & $0.0928(7)$ \\
O6 & $0.9437(2)$ & $0.8692(2)$ & $0.19691(14)$ & $0.1018(9)$ \\
07 & $0.7664(3)$ & $0.8636(4)$ & $0.13739(14)$ & $0.1602(18)$ \\
08 & $0.8442(4)$ & $0.7349(2)$ & $0.1815(3)$ & $0.203(3)$ \\
C1 & $0.07826(19)$ & $0.61579(15)$ & $0.08696(10)$ & $0.0403(5)$ \\
H1A & 0.0360 & 0.6506 & 0.1175 & $0.048^{*}$ \\
H1B & 0.0274 & 0.6165 & 0.0487 & $0.048^{*}$ \\
C2 & $0.2013(2)$ & $0.65943(15)$ & $0.07704(9)$ & $0.0397(5)$ \\
H2A & 0.2422 & 0.6263 & 0.0452 & $0.048^{*}$ \\
H2B & 0.1895 & 0.7228 & 0.0636 & $0.048^{*}$ \\
C3 & $0.40550(18)$ & $0.69357(13)$ & $0.12869(9)$ & $0.0335(4)$
\end{tabular}


Table 2 (continued)

\begin{tabular}{|c|c|c|c|c|}
\hline Atom & $x$ & $y$ & $z$ & $U_{\text {iso }}{ }^{*} / U_{\text {eq }}$ \\
\hline $\mathrm{H} 3$ & 0.4454 & 0.6557 & 0.0982 & $0.040^{*}$ \\
\hline C4 & $0.4040(2)$ & $0.79333(16)$ & $0.10613(12)$ & $0.0501(6)$ \\
\hline $\mathrm{H} 4 \mathrm{~A}$ & 0.3595 & 0.7969 & 0.0667 & $0.075^{\star}$ \\
\hline H4B & 0.4873 & 0.8142 & 0.1024 & $0.075^{\star}$ \\
\hline $\mathrm{H} 4 \mathrm{C}$ & 0.3642 & 0.8316 & 0.1351 & $0.075^{\star}$ \\
\hline C5 & $0.48196(19)$ & $0.68724(14)$ & $0.18932(9)$ & $0.0362(4)$ \\
\hline $\mathrm{H} 5 \mathrm{~A}$ & 0.4328 & 0.7123 & 0.2213 & $0.043^{*}$ \\
\hline H5B & 0.5532 & 0.7272 & 0.1865 & $0.043^{*}$ \\
\hline C6 & $0.52901(17)$ & $0.59238(14)$ & $0.21085(9)$ & $0.0341(4)$ \\
\hline $\mathrm{C} 7$ & $0.59570(19)$ & $0.54337(16)$ & $0.16040(11)$ & $0.0437(5)$ \\
\hline $\mathrm{H} 7 \mathrm{~A}$ & 0.6347 & 0.4886 & 0.1769 & $0.066^{*}$ \\
\hline H7B & 0.6573 & 0.5836 & 0.1451 & $0.066^{*}$ \\
\hline $\mathrm{H} 7 \mathrm{C}$ & 0.5372 & 0.5271 & 0.1273 & $0.066^{*}$ \\
\hline $\mathrm{C} 8$ & $0.62207(19)$ & $0.60658(18)$ & $.26621(11)$ & $0.0472(5)$ \\
\hline $\mathrm{H} 8 \mathrm{~A}$ & 0.5863 & 0.6459 & 0.2959 & $0.071^{*}$ \\
\hline $\mathrm{H} 8 \mathrm{~B}$ & 0.6959 & 0.6346 & 0.2525 & $0.071^{*}$ \\
\hline $\mathrm{H} 8 \mathrm{C}$ & 0.6422 & 0.5480 & 0.2848 & $0.071^{\star}$ \\
\hline C9 & $0.37464(17)$ & $0.55461(15)$ & 28784(9) & $0.0351(4)$ \\
\hline H9A & 0.3634 & 0.6208 & 0.2911 & $0.042^{\star}$ \\
\hline H9B & 0.4340 & 0.5354 & 0.3205 & $0.042^{\star}$ \\
\hline C10 & $0.25306(17)$ & $0.50647(15)$ & $0.29512(9)$ & $0.0340(4)$ \\
\hline $\mathrm{H} 10 \mathrm{~A}$ & 0.2648 & 0.4402 & 0.2940 & $0.041^{*}$ \\
\hline $\mathrm{H} 10 \mathrm{~B}$ & 0.2213 & 0.5225 & 0.3345 & $0.041^{*}$ \\
\hline $\mathrm{C} 11$ & $0.04105(17)$ & .48930(13) & $.24930(9)$ & $0.0316(4)$ \\
\hline H11 & 0.0537 & 0.4224 & 0.2503 & $0.038^{\star}$ \\
\hline $\mathrm{C} 12$ & $-0.0210(2)$ & $0.51756(18)$ & $30777(10)$ & $0.0472(5)$ \\
\hline $\mathrm{H} 12 \mathrm{~A}$ & 0.0344 & 0.5062 & 0.3431 & $0.071^{\star}$ \\
\hline $\mathrm{H} 12 \mathrm{~B}$ & -0.0952 & 0.4823 & 0.3112 & $0.071^{*}$ \\
\hline $\mathrm{H} 12 \mathrm{C}$ & -0.0411 & 0.5820 & 0.3058 & $0.071^{\star}$ \\
\hline C13 & $-0.04677(17)$ & $0.51258(14)$ & $.19390(9)$ & $0.0336(4)$ \\
\hline $\mathrm{H} 13 \mathrm{~A}$ & -0.0513 & 0.5792 & 0.1907 & $0.040^{*}$ \\
\hline H13B & -0.1285 & 0.4910 & 0.2029 & $0.040^{*}$ \\
\hline C14 & $-0.01813(17)$ & $0.47499(14)$ & 13036(9) & $0.0343(4)$ \\
\hline C15 & $-0.1293(2)$ & $0.4952(2)$ & $0.08481(11)$ & $0.0511(6)$ \\
\hline $\mathrm{H} 15 \mathrm{~A}$ & -0.1509 & 0.5592 & 0.0872 & $0.077^{\star}$ \\
\hline H15B & -0.1985 & 0.4581 & 0.0952 & $0.077^{\star}$ \\
\hline $\mathrm{H} 15 \mathrm{C}$ & -0.1078 & 0.4808 & 0.0437 & $0.077^{\star}$ \\
\hline C16 & $0.0032(2)$ & $0.37139(16)$ & $0.13215(12)$ & $0.0488(5)$ \\
\hline $\mathrm{H} 16 \mathrm{~A}$ & 0.0133 & 0.3492 & 0.0911 & $0.073^{\star}$ \\
\hline $\mathrm{H} 16 \mathrm{~B}$ & -0.0667 & 0.3416 & 0.1488 & $0.073^{*}$ \\
\hline $\mathrm{H} 16 \mathrm{C}$ & 0.0763 & 0.3580 & 0.1577 & $0.073^{*}$ \\
\hline C17 & $0.33519(16)$ & $0.38038(13)$ & 10742(8) & $0.0295(4)$ \\
\hline C18 & $0.37371(18)$ & $0.30143(13)$ & $6555(9)$ & $0.0333(4)$ \\
\hline C19 & $0.5133(2)$ & $0.31096(17)$ & $0.05644(12)$ & $0.0490(5)$ \\
\hline $\mathrm{H} 19 \mathrm{~A}$ & 0.5352 & 0.2690 & 0.0241 & $0.059 *$ \\
\hline H19B & 0.5295 & 0.3732 & 0.0426 & $0.059^{*}$ \\
\hline C20 & $0.5958(2)$ & $0.2913(2)$ & $0.11373(15)$ & $0.0651(7)$ \\
\hline $\mathrm{H} 20 \mathrm{~A}$ & 0.5715 & 0.3297 & 0.1469 & $0.098^{*}$ \\
\hline $\mathrm{H} 20 \mathrm{~B}$ & 0.6802 & 0.3042 & 0.1055 & $0.098^{\star}$ \\
\hline $\mathrm{H} 20 \mathrm{C}$ & 0.5880 & 0.2277 & 0.1250 & $0.098^{*}$ \\
\hline C21 & $0.34589(19)$ & $0.20577(14)$ & $0.09103(10)$ & $0.0377(4)$ \\
\hline $\mathrm{H} 21 \mathrm{~A}$ & 0.3917 & 0.1601 & 0.0691 & $0.045^{*}$ \\
\hline $\mathrm{H} 21 \mathrm{~B}$ & 0.3745 & 0.2033 & 0.1341 & $0.045^{\star}$ \\
\hline $\mathrm{C} 22$ & $0.3028(2)$ & $0.31099(16)$ & $0.00260(9)$ & $0.0407(5)$ \\
\hline $\mathrm{H} 22 \mathrm{~A}$ & 0.3076 & 0.3744 & -0.0113 & $0.049^{*}$ \\
\hline $\mathrm{H} 22 \mathrm{~B}$ & 0.3413 & 0.2722 & -0.0272 & 0.049 * \\
\hline
\end{tabular}

Table 2 (continued)

\begin{tabular}{lrrrr}
\hline Atom & $\boldsymbol{x}$ & $\boldsymbol{y}$ & $\boldsymbol{z}$ & $\boldsymbol{U}_{\text {iso }} \boldsymbol{U}_{\text {eq }}$ \\
\hline $\mathrm{C} 23$ & $0.1700(2)$ & $0.19155(14)$ & $0.02419(9)$ & $0.0377(4)$ \\
$\mathrm{H} 23$ & 0.2203 & 0.1543 & -0.0024 & $0.045^{*}$ \\
$\mathrm{C} 24$ & $0.0396(2)$ & $0.15631(14)$ & $0.01918(9)$ & $0.0379(4)$ \\
$\mathrm{C} 25$ & $-0.0079(2)$ & $0.11880(17)$ & $-0.03608(11)$ & $0.0491(5)$ \\
H25 & 0.0396 & 0.1195 & -0.0704 & $0.059^{*}$ \\
$\mathrm{C} 26$ & $-0.1247(2)$ & $0.08056(18)$ & $-0.04062(12)$ & $0.0551(6)$ \\
H26 & -0.1558 & 0.0556 & -0.0778 & $0.066^{*}$ \\
$\mathrm{C} 27$ & $-0.1944(2)$ & $0.07955(15)$ & $0.00986(12)$ & $0.0462(5)$ \\
C28 & $-0.1507(2)$ & $0.11636(17)$ & $0.06520(12)$ & $0.0497(5)$ \\
H28 & -0.1990 & 0.1158 & 0.0992 & $0.060^{*}$ \\
C29 & $-0.0330(2)$ & $0.15441(16)$ & $0.06931(10)$ & $0.0445(5)$ \\
H29 & -0.0025 & 0.1792 & 0.1066 & $0.053^{*}$ \\
\hline
\end{tabular}

amount of water. After a few days, blue crystals were separated in $\sim 32 \%$ yield. Anal. Calcd. for $\mathrm{C}_{29} \mathrm{H}_{52} \mathrm{Cl}_{2} \mathrm{~N}_{4} \mathrm{NiO}_{9}$ : C, 47.69; H, 7.17; N, 7.67\%. Found: C, 47.25; H, 7.51; N, 7.89\%. IR data (cm $\left.{ }^{-1}, \mathrm{KBr}\right): 3456(\mathrm{~m}), 3209(\mathrm{~s}), 2962(\mathrm{~m}), 1545(\mathrm{~s}), 1450(\mathrm{~s})$, 1090(s).

\section{Experimental details}

All the hydrogen atoms of the ligands were placed in calculated positions with fixed isotropic thermal parameters and included in the final stage of refinement. The $U_{\text {iso }}(\mathrm{H})$ values of methyl groups were set to $1.5 U_{\text {eq }}(\mathrm{C})$ and the $U_{\text {iso }}$ values of all other hydrogen atoms were set to $1.2 U_{\text {eq }}(C, N)$.

\section{Discussion}

Ketal compounds are subjects of great interest because they are usually used as a protection of carbonyl or synthetic intermediates [4], and have been widely applied in fragrance and flavors as well as a new type of spices.

X-ray crystal structural analysis reveals that the title compound contains one complex cation [ $\left.\mathrm{Ni}\left(\mathrm{C}_{16} \mathrm{H}_{36} \mathrm{~N}_{4}\right)\left(\mathrm{C}_{13} \mathrm{H}_{14} \mathrm{O}_{4} \mathrm{Cl}\right)\right]^{+}$, one anion $\left[\mathrm{ClO}_{4}\right]^{-}$, and one water molecule. Each Ni(II) ion lies on a general position of the monoclinic space group and is coordinated by four macrocyclic nitrogen atoms of $\mathrm{L}$ in a folded conformation and two carboxylate oxygen atoms of the anionic 4-(chlorophenyl)5-ethyl-1,3-dioxane-5-carboxylato ligand. The $\mathrm{Ni}-\mathrm{N}$ bond lengths $[2.090(6)-2.131(6) \AA]$ are slightly shorter than the Ni-O bond lengths [2.120(4) and 2.261(4) Å]. Neighbouring cations and anions are discrete, connected to each other through hydrogen bonds between the carboxylate oxygen atom of 4-(chlorophenyl)-5-ethyl-1,3-dioxane-5-carboxylato ligand and water and nitrogen atom of adjacent cation, forming a one-dimensional chain.

Acknowledgements: This work was financially Supported by the NSFC (51772091), the Scientific Research Fund of 
Hunan Education Department (17A081) and the Science and Technology Innovative plan of Hunan (2017).

\section{References}

1. Bruker: APEX3, SAINT-Plus, XPREP. Bruker AXS Inc., Madison, Wisconsin, USA (2016).
2. Sheldrick, G. M.: SHELXT - Integrated space-group and crystal-structure determination. Acta Crystallogr. A71 (2015) 3-8.

3. Sheldrick, G. M.: Crystal structure refinement with SHELXL. Acta Crystallogr. C71 (2015) 3-8.

4. Ono, D.; Yamamura, S.; Nakamura, M.: Preparation and properties of bis(sodium sulfate) types of cleavable surfactants derived from diethyl tartrate. J. Oleo. Sci. 54 (2005) 51-57. 